\title{
Durch Kombinationen noch mehr erreichen bei metastasiertem Melanom
}

\author{
Die Immuncheckpointinhibition sowie die zielgerichtete Therapie \\ mit BRAF- und MEK-Blockade haben die Überlebensaussichten für \\ therapienaive Patienten mit metastasiertem BRAF-mutiertem \\ inoperablem Melanom enorm verbessert. Und es geht offenbar \\ noch mehr.
}

- ffizient, so führte Ralf Gutzmer, Hannover, aus, seien bei BRAFmutierten Tumoren nachgewiesenermaßen beide Therapieansätze, auch wenn sie sich hinsichtlich ihrer Applikation und Wirkmechanismen grundsätzlich unterscheiden: Während sich die

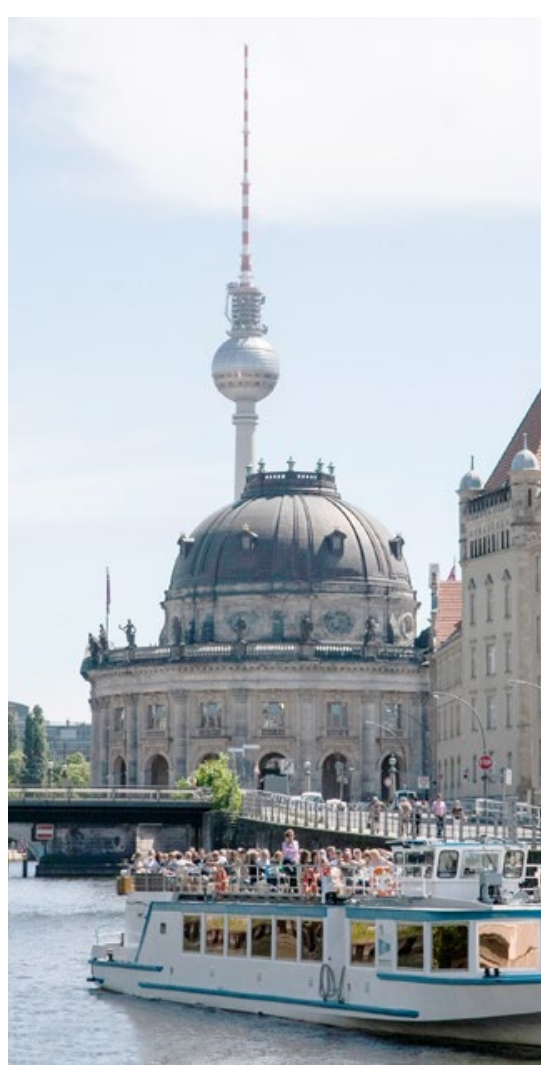

Gute Aussichten - für die DDG-Teilnehmer in Berlin und für Melanompatienten. zielgerichtete Therapie gezielt auf die Mutation richtet, oral verabreicht werden kann und ihre Wirkung unmittelbar in den Krebszellen entfaltet, zielen die Immuncheckpointinhibitoren auf das Immunsystem, wirken nicht in, sondern auf den Zellen und werden intravenös verabreicht. In der Regel tritt bei zielgerichteter Therapie der gewünschte Effekt schneller ein als bei Immuncheckpointblockade, die Ansprechraten auf die Behandlung liegen bei $70 \%$. Zwar werden bei der Immuntherapie nur Ansprechraten von $40-60 \%$ erreicht, jedoch ist die Wirkdauer mit im Median mehr als 24 Monaten nahezu doppelt so hoch wie bei zielgerichteter Therapie mit median circa 13 Monaten. Die Nebenwirkungen sind bei zielgerichteter Therapie dosisabhängig und in der Regel reversibel, bei der Immuncheckpointblockade handelt es sich vorrangig um Entzündungen und teils irreversible Schäden.

\section{Bessere Überlebensdaten bei Kombination}

Welcher Patient sollte welcher Therapie zugeführt werden? Diese Entscheidung kann bislang nur anhand „weicher" Kriterien getroffen werden, etwa der Bevorzugung einer bestimmten Applikationsform durch den Patienten oder der Art von Komorbiditäten - valide Marker für die Entscheidung pro oder kontra zielgerichtete bzw. Immuntherapie existieren derzeit nicht. Kombinationstherapien erhöhen die Gesamtüberlebensraten deutlich. So lassen sich bei einer Kombi- nation von BRAF- und MEK-Inhibitor (z.B. Dabrafenib plus Trametinib, Vemurafenib plus Cobimetinib) Gesamtüberlebensraten nach 24 Monaten von $50-55 \%$ erreichen, bei einer Kombination von Immuncheckpointinhibitoren (CTLA-4-Hemmer Ipilimumab plus PD-1-Blocker Nivolumab) sogar 55$65 \%$.

\section{Zielgerichtete plus Immuntherapie erfolgversprechend}

Gutzmer zufolge ist zu vermuten, dass sich das Langzeitüberleben der Patienten durch eine Kombination der zielgerichteten Therapien mit den Immuntherapeutika noch erheblich verbessern lässt. Erste Studienansätze dazu gibt es, etwa die Phase-II-Studie ImmunoCobiVem. An dieser nehmen unvorbehandelte erwachsene Patienten mit nicht-resektablem metastasiertem BRAFv600-mutiertem Melanom (Stadium IIIc/IV) und ausreichender Leber- und Nierenfunktion teil (ClinicalTrials.gov Identifier: NCT02902029). Sie erhalten zunächst drei Monate lang den BRAF-Inhibitor Vemurafenib und den MEK-Inhibitor Cobimetinib. Anschließend bekommt die eine Patientengruppe weiterhin Vemurafenib und Cobimetinib, die andere wird mit dem PD-L1(„programmed cell death-ligand 1“)-Inhibitor Atezolizumab behandelt. Bei Progression der Erkrankung ist ein Crossover in den jeweils anderen Arm vorgesehen.

In der Phase-III-Studie TRILOGY wird die Kombination aus Vemurafenib und Cobimetinib randomisiert plus Placebo oder Atezolizumab getestet (ClinicalTrials.gov Identifier: NCT02908672).

Kathrin von Kieseritzky

Bericht von der 49. Haupttagung der Deutschen Dermatologischen Gesellschaft vom 26. bis 29. April 2017 in Berlin 